\title{
EP-182
}

\section{How mutations in pancreatic cancer are treated- Is surgery the only option?}

\author{
Tanvi GUPTA, Wen-Pin SU*
}

Institute of Clinical Medicine, National Cheng Kung University, Tainan, Taiwan

Introduction: Pancreatic cancer is a lethal disease having a worst five-year overall survival rate of $2 \%$ to $9 \%$ compared to other cancer types. The occurrence of pancreatic cancer is rising due to numerous factors such as intake of alcohol, smoking, obesity, dietary factors, and Helicobacter pylori infection. Most of the pancreatic cancers have gene mutations includes BRCA1, BRCA2, CDKN2A, ATM, PALB2, TP53, SMAD4, KRAS, etc. and from these BRCA1/2 have found to be more prevalent among the patients.

Methods: The treatment options for the pancreatic cancer are Pancreatico-duodenectomy includes pre-operative biliary drainage, an anastomotic technique, invasive surgery, vascular resection, and adjuvant as well as neo- adjuvant treatment. The metastatic cancer can be taken care by control of symptoms, management of jaundice and with favoured chemotherapy- mFOLFIRINOX with 5- fluorouracil.

Results: The patients treated with adjuvant gemcitabine after the surgery showed five-year overall survival rate of $20.7 \%$ against the surgery alone with $10.4 \%$. The dual therapy of gemcitabine and capecitabine showed the median overall survival to be 28 months compared to gemcitabine alone to be 25.5 months. The other chemotherapy way by mFOLFIRINOX compared with gemcitabine alone showed 21.6 months versus 12.8 months for the disease free survival and 54.4 months versus 35 months overall survival.

Conclusions: As we know pancreatic cancer turns out to be fatal with poor overall survival rate, so there is need to explore more treatment options. Many studies are ongoing to find the standard of care for patients whether it would be or not be combinations of cytotoxic with targeted. 